\title{
SALUD
}

\section{Presentación de un instrumento para evaluación de impacto a la salud por exposición a neurotóxicos en población trabajadora. Estudio preliminar}

\author{
Laura Emilce Flores Rodríguez
}

\begin{abstract}
Resumen
Introducción: Existen varias ocupaciones que conllevan la exposición a neurotóxicos, por el uso en las industrias de solventes, metales y plaguicidas, pudiendo producir cambios importantes como alteraciones sensoriales, motoras, alteraciones en la capacidad de aprendizaje, memoria, emocionales entre otros. En nuestro país existen pocos datos y estudios en el área, además se cuenta con diversas ocupaciones que involucran el uso de estas sustancias. Una de ellas es la minería artesanal de oro, considerado uno de los procesos extractivos que genera mayor cantidad de problemas ambientales, y a la salud humana, en parte por la utilización de mercurio; pues se calcula que, por cada gramo de oro extraído, se pueden utilizar desde 7 a 30 gramos de mercurio, de acuerdo a la técnica utilizada. El uso inadecuado del mercurio, principalmente en la minería del oro, además de producir contaminación ambiental significativa, tiene efectos neurotóxicos y sistémicos tanto en exposiciones ocupacionales como ambientales.
\end{abstract}

Objetivo: Diseñar un instrumento que permita evaluar el impacto en salud por exposición a neurotóxicos específicamente en el uso del mercurio en la minería artesanal de oro.

Material y Método: Se realizado una búsqueda exhaustiva de la literatura buscando estudios e investigaciones de evaluaciones de signos y síntomas neurotóxicos para tóxicos ocupacionales y ambientales, en especial aquellos vinculados al uso del mercurio. Se recalca que este es el primer paso para la evaluación de impacto en salud.

Resultados: Se diseñó el instrumento conteniendo los siguientes ítems: a) Historia clínico Toxicología Laboral de Nelson Albiano, Medicina Laboral de Joseph Ladou, Enciclopedia de la OIT, Instituto de Seguridad e Higiene del Trabajo de España. Se agregaron preguntas sobre consumo de pescado. B) Para la evaluación de signos y síntomas neurotóxicos se revisaron el cuestionario de Síntomas neurotóxicos Q 16, el Mini Mental test de Caban y estudio

1 Investigación para el Desarrollo Vitalitas.

E-mail: floreslaurapy@yahoo.com

DOI: 10.26885/rcei.foro.2018.128 
tremográfico. El Q16 contiene dieciséis preguntas validadas acerca de síntomas de neurotoxicidad. La comprensión de las preguntas fue investigada por médicos y psicólogos. La confiabilidad fue evaluada realizando procedimientos de prueba y reprueba. La validez fue evaluada investigando el poder de las preguntas o discriminando. El Mini Mental test de 30 preguntas agrupadas en 10 secciones evalúa: Orientación espacio temporal Capacidad de atención, concentración y memoria Capacidad de abstracción (cálculo), Capacidad de lenguaje y percepción visual-espacial. Capacidad para seguir instrucciones básicas. Para la evaluación de la vigilancia biológica de la exposición se utilizará el dosaje de mercurio en orina de 24 horas.

Conclusiones: Con la aplicación del instrumento se evaluará síntomas neurotóxicos en la población expuesta, posteriormente se asociará con los valores de mercurio en orina de 24 horas.

Palabras clave: neurotoxicidad, uso del mercurio, minería de oro.

\section{Referencias}

Anger, K. (2003). Neurobehavioral tests and systems to assess neurotoxic exposures in the workplace and community. Occupational and Environmental Medicine, 60(7), p. 531-8.

Diaz-Arriaga, F. (2014). Mercurio en la minería del oro: impacto en las fuentes hídricas destinadas para consumo humano. Rev. salud pública, 16(6), p. 947-957.

Lebel, J., Mergler, D., Lucotte, M. (1996). Evidence of Early Nervous Systems Dysfunction in Amazonian Populations Exposed to Low-Levels of Methylmercury. Neurotoxicology, (17), p. 157-168.

Ministerio del Medio Ambiente de Japón. (2013). Enseñanzas de la Enfermedad de Minamata y el Manejo del Mercurio en Japón. 